\title{
Encarceramento do intestino delgado em forame epiploico em equinos com hábito de aerofagia: relato de caso
}

\author{
[Entrapment of the small intestine in the epiploic foramen in crib-biting horses: case report] \\ J.M. Alonso, M.J. Watanabe, C.A. Rodrigues, A.L.G. Alves, C.M. Ramos, \\ G.S. Rosa, C.A. Hussni*
}

Universidade Estadual Paulista - Botucatu, SP

\begin{abstract}
RESUMO
São relatados quatro casos de encarceramento de forame epiploico (EFE), entre os casos: três machos e uma fêmea; dois da raça Quarto de Milha, um SRD e um Brasileiro de Hipismo; idade média de 11 anos; altura média de cernelha de $160,5 \mathrm{~cm}$; massa corporal média de $465 \mathrm{~kg}$. Todos os animais possuíam o hábito de aerofagia. A presença de refluxo enterogástrico e intestino delgado distendido à palpação transretal foi variável na dependência do segmento intestinal aprisionado. À laparotomia exploratória constataram-se: dois casos de aprisionamento jejunoileal; um de jejuno; e o outro de íleo. Todos os animais apresentaram EFE da esquerda para a direita, representando o sentido horário. Apenas o animal que apresentava o íleo aprisionado sobreviveu; os demais foram submetidos à eutanásia no período transoperatório devido à extensa isquemia intestinal e às lesões vasculares decorrentes da manobra de redução. A aerofagia é o fator de risco que apresenta maior correlação positiva com o EFE, fato corroborado pelos casos apresentados. Apesar de ser descrito um bom prognóstico para o EFE na literatura, foi obtido um desfecho ruim entre os casos atendidos, e salienta-se que a extensão da lesão e o sangramento da veia porta no transoperatório contribuíram para esse resultado.
\end{abstract}

Palavras-chave: aerofagia, cólica, cavalos, forame epiploico

\begin{abstract}
Four cases of epiploic foramen entrapment (EFE) are reported. Among the cases, three males and one female, two Quarter Horses, one mixed breed and one Brazilian Equestrian, aged between 3 to 20 years, mean 11 years. The average height was $160.5 \mathrm{~cm}$ and $465 \mathrm{~kg}$. All horses had a previous record of cribbiting habit. The presence of enterogastric reflux and small intestine distended loops in transrectal palpation was variable in dependence of the trapped segment. In surgical exploration were identified two cases of jejunum and ileum entrapped, one of the ileum and one of jejunum. All animals showed EFE from left to right. The horse that had trapped the ileum has survived. The others were euthanized due to the extent of injury or rupture of large vessels during surgical reduction. Although there are many notes of possible risk factors for EFE occurrence, cribbing is the risk factor that has the highest positive correlation with EFE. This appointment was confirmed by the cases presented. Despite being described a good prognosis for the EFE, a bad outcome was achieved among the cases, and stresses that the extent of the injury and bleeding from the portal vein contributed to this outcome.
\end{abstract}

Keywords: colic, cribbing, epiploic foramen, horses

\section{INTRODUÇÃO}

O encarceramento no forame epiploico (EFE) é a segundo processo mais comum de lesão estrangulante de intestino em equinos submetidos à laparotomia, representando 5 a

Recebido em 9 de abril de 2017

Aceito em 13 de junho de 2018

*Autor para correspondência (corresponding author)

E-mail: cahussni@fmvz.unesp.br
$7,7 \%$ dos casos cirúrgicos, 14 a $19 \%$ das lesões de intestino delgado e 2,1 a 8,4\% entre todas as cólicas (Archer et al., 2004ab; Freeman, 2012).

O forame epiploico apresenta 4-6cm de comprimento e está localizado na porção craniodorsal direita do abdômen; é delimitado 
por estruturas nobres, como pâncreas, fígado, veias porta e cava caudal, estômago e duodeno (Freeman e Pearn, 2015; Bergen et al., 2015). O forame epiploico comunica a bursa omental com a cavidade peritoneal (Vachon e Fischer, 1995; Schmid, 1997; Mueller et al., 2008; Freeman, 2012; Freeman e Pearn, 2015).

Por muitos anos, a ocorrência do EFE foi associada à retração do lobo caudato do fígado decorrente do avançar da idade, o que proporcionaria consequente aumento do forame epiploico, já que o lobo caudato é um delimitante anatômico dessa estrutura (Wheat, 1972; Livesey et al., 1991; Engelbert et al., 1993). Entretanto, atualmente essa teoria foi descartada, e estudos recentes demonstram uma correlação positiva entre o hábito de aerofagia e o EFE, com uma taxa de propensão de oito a 35 vezes maior em animais que possuem esse hábito (Archer et al., 2004a,b). Sugere-se que, durante o movimento da caixa torácica para a aerofagia, ocorre uma abertura da bursa omental, devido à tração do estômago através do ligamento gastrofrênico, que une o estômago ao diafragma (Freeman e Pearn, 2015), além de o movimento causar uma mudança na pressão intra-abdominal, que pode contribuir para a expansão do forame e insinuação do intestino delgado através dele (Albanese et al., 2013).

Este relato tem por objetivo descrever quatro casos de encarceramento de intestino delgado em forame epiploico, relacionando os casos com os seus principais fatores de risco, em especial o hábito de aerofagia.

\section{RELATO DOS CASOS}

No período de maio a agosto de 2015, foram atendidos no Hospital Veterinário da FMVZ Unesp, Campus de Botucatu, quatro equinos adultos, com idade entre três e 20 anos, com sinais de desconforto abdominal agudo. Todos os animais foram encaminhados para a laparotomia exploratória e diagnosticados no transoperatório com encarceramento de diferentes porções de intestino delgado através do forame epiploico.

Caso 1: macho, de nove anos de idade, raça Brasileiro de Hipismo, 500kg de massa corporal e altura de cernelha de $170 \mathrm{~cm}$, com histórico de aerofagia, apresentando sinais de dor abdominal moderada havia aproximadamente quatro horas.
Ao exame físico, o animal apresentava frequência cardíaca (FC) de 80bpm, e tempo de preenchimento capilar de três segundos. À sondagem nasogástrica, verificaram-se $8 \mathrm{~L}$ de refluxo espontâneo com pH 7. Ao hemograma, observou-se fibrinogênio de $400 \mathrm{mg} / \mathrm{dL}, 11.400$ leucócitos/ $\mu \mathrm{L}$, com 9.900 neutrófilos. O líquido peritoneal foi coletado e encaminhado para análise laboratorial, apresentando coloração alaranjada, aspecto turvo, pH 8 e 2.050 células nucleadas/ $\mu \mathrm{L}$. À palpação transretal, verificou-se deslocamento de cólon maior que, associado à piora dos sinais clínicos, resultou em indicação cirúrgica. Realizada a laparotomia exploratória, constatou-se encarceramento em sentido da esquerda para a direita de parte do jejuno e íleo através do forame epiploico, com presença de conteúdo alimentar compactado em seu interior e distensão gasosa de jejuno. $\mathrm{O}$ animal foi submetido à eutanásia devido à ruptura dos grandes vasos durante a tentativa de redução do encarceramento.

Caso 2: macho, 12 anos de idade, sem raça definida, peso $420 \mathrm{~kg}$, altura de cernelha de $165 \mathrm{~cm}$, com histórico de aerofagia e cólicas recorrentes. $\mathrm{O}$ animal foi encaminhado ao Hospital Veterinário 12 horas após o início de dor abdominal intensa. Ao exame físico, apresentava aumento de FC (80bpm), mucosas ictéricas com halo toxêmico e desidratação. À sondagem nasogástrica, não se verificou presença de refluxo espontâneo. Ao hemograma, identificou-se fibrinogênio de $200 \mathrm{mg} / \mathrm{dL}$ e 6.000 leucócitos $/ \mu \mathrm{L}$. O líquido peritoneal apresentou aspecto avermelhado, turvo e contagem de 20.947 células nucleadas. O animal foi encaminhado à cirurgia devido à dor refratária. $\mathrm{Na}$ laparotomia exploratória, constatou-se encarceramento de aproximadamente três metros de jejuno através do forame epiploico, no sentido da esquerda para a direita. O segmento encarcerado apresentava-se isquêmico, cianótico e em atonia. $\mathrm{O}$ animal foi submetido à eutanásia devido à ruptura dos grandes vasos e à decorrente hemorragia cavitária adominal na tentativa de redução do encarceramento.

Caso 3: fêmea, 20 anos de idade, raça Quarto de Milha, peso $470 \mathrm{~kg}$ e altura de cernelha de $152 \mathrm{~cm}$, com histórico de aerofagia. O animal foi atendido por médico veterinário na propriedade e encaminhado ao Hospital Veterinário aproximadamente seis horas após a observação 
de sinais de dor abdominal intensa. Ao exame físico, apresentou aumento de FC (120bpm), congestão de mucosas e desidratação intensa. Não houve presença de refluxo enterogástrico. Ao hemograma, fibrinogênio de $400 \mathrm{mg} / \mathrm{dL}$, 12.100 leucócitos/ $\mu \mathrm{L}$, com 10.300 neutrófilos, presença de neutrófilos tóxicos com granulações citoplasmáticas. A coleta de líquido peritoneal foi improdutiva. Ao exame ultrassonográfico, observou-se distensão de intestino delgado em toda a cavidade abdominal, e os segmentos distendidos e localizados na região abdominal epigástrica direita e esquerda apresentavam espessamento da parede intestinal $(0,8 \mathrm{~mm})$. À palpação transretal, observou-se presença de intestino delgado distendido e projetado na pelve. $\mathrm{O}$ animal foi encaminhado para laparotomia exploratória. Durante a exploração sistemática do abdômen, constatou-se EFE de grande extensão do jejuno no sentido da esquerda para a direita e lesão isquêmica de jejuno e íleo. O animal foi submetido à eutanásia devido ao comprometimento vascular de grande porção do intestino delgado.

Caso 4: macho, três anos de idade, raça Quarto de Milha, peso $470 \mathrm{~kg}$ e altura de cernelha de $155 \mathrm{~cm}$, com histórico de aerofagia. $\mathrm{O}$ animal foi atendido por médico veterinário na propriedade, sendo encaminhado ao Hospital Veterinário aproximadamente 12 horas após o início dos sinais de dor abdominal moderada. Ao exame físico, apresentava aumento de FC (80bpm), desidratação moderada, mucosas róseas e ausência de refluxo enterogástrico espontâneo. Ao hemograma, foram obtidos valores de leucócitos de $12.100 / \mu \mathrm{L}$, com 9.100 neutrófilos. Não foi possível coletar líquido peritoneal. O animal foi encaminhado para laparotomia exploratória devido à presença de alças de intestino delgado distendidas e de dor refratária à administração de analgésicos. Durante o procedimento cirúrgico, foi observado encarceramento de cerca de $30 \mathrm{~cm}$ de íleo através do forame epiploico, no sentido da esquerda para a direita, com grande distensão gasosa de jejuno. Foi possível realizar a redução do encarceramento mediante tração delicada, no sentido da direita para a esquerda das alças encarceradas, após a aspiração do máximo possível de gás contido nos segmentos adjacentes ao encarceramento. $\mathrm{O}$ animal apresentou um pósoperatório sem complicações e recebeu alta 15 dias após o procedimento cirúrgico. Após 60 dias, foi possível saber, por meio de contato telefônico, que o animal se manteve estável, sem complicações ou recidiva da lesão.

\section{DISCUSSÃO E CONCLUSÃO}

Quanto às raças relacionadas, houve variação, com a ocorrência em dois animais Quarto de Milha, um SRD e um Brasileiro de Hipismo. É descrito que os animais da raça Puro Sangue Inglês (PSI) têm maior predisposição ao EFE (Vachon e Fischer, 1995; Archer et al., 2004a). Entretanto, atualmente essa predisposição aparenta ter maior relação com o hábito de aerofagia, enfermidade cuja predisposição é maior em animais da raça PSI (Wickens e Heleski, 2010), e com a altura do animal tomada pela cernelha, do que propriamente com a raça (Archer et al., 2008). Entre os animais relatados, dois apresentavam estatura maior, com 170 e $165 \mathrm{~cm}$ de altura, e dois apresentavam estatura mediana, com 152 e $155 \mathrm{~cm}$ de altura. Aparentemente, o EFE parece mais prevalente em machos (Archer et al., 2004b), predisposição observada também entre os animais relatados, sendo três machos e uma fêmea.

É descrita maior prevalência da afecção $(73,5 \%)$ entre os meses de novembro e março na América do Norte (Archer et al., 2004a). No presente estudo, os casos relatados ocorreram entre maio e agosto; em ambos os locais, no período de transição entre o outono e o inverno, e a variação segue a sazonalidade continental a que se refere.

Apesar de haver correlação positiva entre o EFE e o histórico de cólicas anteriores (Archer et al., 2008), somente um dos animais apresentou histórico de cólica nos 12 meses anteriores.

Por muitos anos, a ocorrência do EFE foi relacionada à idade do animal, sendo descrito que animais idosos apresentam retração do lobo hepático caudato, o que poderia favorecer o EFE devido à maior amplitude do forame epiploico secundária a essa retração (Wheat, 1972; Livesey et al., 1991; Engelbert et al., 1993). No entanto, essa teoria não foi corroborada por estudos subsequentes, tornando-se obsoleta (Schmid, 1997; Freeman e Schaeffer, 2001; Archer et al., 2004a). Em um estudo desenvolvido por Schmid (1997), foi realizada a mensuração post mortem do forame de 15 animais de diferentes idades, a 
qual constatou não haver diferença do tamanho do forame com o avançar da idade.

Existem relatos que correlacionam positivamente a aerofagia e a ocorrência do EFE (Archer et al., 2004a; Archer et al., 2008). Entre os animais atendidos, apesar das variações de raça, sexo e idade, todos eles apresentavam em comum o hábito de aerofagia. Durante o movimento da caixa torácica para a aerofagia, sugere-se que ocorra uma abertura da bursa omental devido à tração do estômago através do ligamento gastrofrênico, que une o estômago ao diafragma (Freeman e Pearn, 2015), e, ainda, que a aerofagia cause uma mudança na pressão intraabdominal, que pode contribuir para a expansão do forame e insinuação do intestino delgado através dele (Albanese et al., 2013).

A correlação entre a aerofagia e a síndrome cólica já era previamente descrita nos casos de cólicas gasosas, cólicas decorrentes de problemas dentários devido ao desgaste excessivo dos dentes, pois a mastigação irregular predisporia à ingestão de partículas grandes, à gastrite e a úlceras em animais jovens (Archer et al., 2004a). Entretanto, a associação da aerofagia com o EFE foi inicialmente descrita por Archer et al. (2004), que, em um estudo retrospectivo de 11 anos, computaram o atendimento de 68 casos de EFE em dois hospitais veterinários. Nesses hospitais, foi detectado que $68 \%$ dos animais que apresentaram EFE tinham histórico de aerofagia, comparados com somente $6 \%$ nas outras lesões estrangulativas de intestino delgado.

A correlação da aerofagia com o EFE, descrita na literatura internacional, não é claramente explanada no Brasil (Archer et al., 2004a; Archer et al., 2008), que apresenta estudos escassos e sem a associação das alterações apresentadas. É descrito um único relato em um animal com aerofagia e lesão hepática (Canisso e Souza, 2009). Todavia, os dados epidemiológicos associados ao risco de desenvolvimento dessa afecção, como época do ano, sexo, idade e raça, são extrapolados da literatura internacional, o que pode representar uma lacuna no traçado epidemiológico para o cenário nacional devido às diferenças geográficas, climáticas e meteorológicas.

Os animais com EFE apresentaram sinais clínicos compatíveis com lesão estrangulativa de intestino delgado, como elevação da frequência cardíaca (média de 90bpm) e elevação do tempo de preenchimento capilar (média de três segundos), além de temperatura retal média de $37,5^{\circ} \mathrm{C}$ e ausência de borborigmos intestinais. A presença de refluxo enterogástrico foi observada em somente um animal, possivelmente devido ao fato de o refluxo ser tardio na dependência do segmento de intestino delgado acometido (Archer et al., 2008). Apesar de alguns animais apresentarem distensão de segmentos de intestino delgado, não foi possível detectar tal alteração durante a palpação transretal devido ao fato de o forame epiploico ser localizado na região cranial do abdômen e haver pouco segmento de intestino delgado acometido. Dessa forma, salienta-se a importância do exame ultrassonográfico (Vachon e Fischer, 1995), que permite identificar a distensão intestinal, frequentemente de difícil diagnóstico à palpação transretal, além de seus achados sugerirem fortemente a ocorrência de EFE devido à localização da distensão e ao espessamento de parede das alças.

Quanto ao hemograma dos animais, três apresentaram número normal de leucócitos totais, porém com neutrofilia, e um dos animais estava com leucopenia. Era esperado que os neutrófilos do sangue, distribuídos nos compartimentos circulantes e marginais na decorrência dos estímulos quimiotáxicos relacionados ao processo inflamatório, apresentassem marginação e migração para as alças intestinais comprometidas ou para a cavidade abdominal, com imediata diminuição do seu número na circulação (Zinkl e Mahendra, 1997). Possivelmente, por meio de estímulos ao eixo hipotálamo-pituitária-adrenal, houve liberação de corticosteroides pelo córtex da adrenal, aumentando a concentração de corticosteroides endógenos circulantes, em resposta ao estresse fisiológico ou à doença, o que pode ter resultado em alterações na contagem diferencial de leucócitos, destacandose a neutrofilia (Breider, 1993).

Devido ao grau de desidratação apresentado pelos animais, a coleta de líquido peritoneal foi improdutiva em alguns casos. Dessa forma, a discussão dos resultados obtidos fica prejudicada. É descrito por Vachon e Fischer (1995) que, nos casos de EFE, ocorre um aumento na contagem de células nucleadas, 
entretanto, em dois dos animais com EFE, obtiveram-se os valores de 2.050 células $/ \mathrm{mL}$ e 20.947 células $/ \mathrm{mL}$, valores pouco expressivos para o grau de comprometimento intestinal e consequente óbito. Entretanto, Livesey et al (1991) relatam que, no EFE, é obtido um líquido peritoneal avermelhado, porém com baixa contagem de células nucleadas. Sugere-se que o líquido peritoneal alterado se acumulou inicialmente na bursa omental, caracterizando uma peritonite localizada, com sequestro de fluido pelo omento, conforme previamente descrito por Mueller et al. (2008) e Freeman (2012)

Archer et al. (2004b) descreveram 71 casos de $\mathrm{EFE}$, entre os quais 69 ocorreram da esquerda para a direita e apenas dois da direita para a esquerda. Nesses 71 casos, em 17 deles somente o íleo estava aprisionado, jejuno e íleo em 30 casos, e jejuno sozinho em 24 casos. Nos quatro casos relatados no presente estudo, todos ocorreram da esquerda para a direita. Em um deles, somente o íleo estava aprisionado; em dois, jejuno e íleo; e em um, apenas jejuno. Os quatro casos ora relatados seguem a mesma proporção dos segmentos intestinais acometidos no aprisionamento, em semelhança ao exposto por Archer et al. (2004b).

Dos 71 casos descritos por Archer et al. (2004b), oito foram submetidos à eutanásia no período transcirúrgico. Destes, um apresentou ruptura gástrica, três apresentaram grande extensão de intestino inviável, quatro apresentaram hemorragia intra-abdominal intensa, sendo essas hemorragias advindas da veia porta em um caso, de um vaso mesentérico profundo em outro, e nos demais de origem não identificada. Dos quatro casos abordados, a extensão do segmento intestinal foi indicativa de eutanásia em dois dos animais, tendo um desses apresentado ainda hemorragia intensa. A hemorragia foi, portanto, identificada em dois dos animais e, mesmo sem ter a origem determinada, foi atribuída aos grandes vasos adjacentes à bursa omental, provavelmente proveniente da veia porta. Segundo Livesey et al. (2015), a veia porta é o foco mais frequente de hemorragia associada ao EFE, e não há possibilidade de correção, tendo em vista a impossibilidade de acesso cirúrgico.
As taxas de sobrevivência, em curto prazo, variam de 66 a $74 \%$ e, em longo prazo, de 79 a 95\% (Vachon e Fischer, 1995; Archer et al., 2004b; Freeman e Schaeffer, 2001; Archer et al., 2011). Entre os animais atendidos, apenas um teve prognóstico bom, com alta após 15 dias e evolução favorável após 60 dias, mediante informações via contato telefônico. A viabilidade intestinal do segmento aprisionado, bem como a pequena extensão aprisionada, permitiu menor manipulação, e não requereu enterectomia, o que possivelmente contribuiu para a sobrevivência do animal 4. Em estudo retrospectivo desenvolvido por Archer et al. (2011), 63 casos de encarceramento epiploico foram avaliados quanto à taxa de sobrevida pós-operatória, e observou-se que o íleo pós-operatório apresenta correlação positiva com a diminuição da sobrevida nessa enfermidade, bem como a frequente necessidade transoperatória de ressecção intestinal. Possivelmente, a intensa manipulação cirúrgica para correção do aprisionamento possa favorecer o íleo.

O resultado ruim obtido na maioria dos casos foi decorrente da extensa lesão do intestino delgado, do encaminhamento tardio e da frequente ruptura dos vasos na tentativa de redução, mesmo quando utilizadas técnicas para esvaziamento do gás e do conteúdo intestinal, associada à tração leve e delicada.

Apesar de haver diversos apontamentos de possíveis fatores de risco para a ocorrência do EFE, a aerofagia é o fator de risco que apresenta maior correlação positiva com a ocorrência da enfermidade, o que é corroborado nesses casos apresentados.

\section{REFERÊNCIAS}

ALBANESE, V.; MUNSTERMAN, A.S.; DEGRAVES, F.J.; HANSON, R.R. Evaluation of intra-abdominal pressure in horses that crib. Vet. Surg., v.42, p.658-662, 2013.

ARCHER, D.C.; FREEMAN, D.E.; DOYLE, A.J.; PROUDMAN, C.J.; EDWARDS, G.B. Association between cribbing and entrapment of the small intestine in the epiploic foramen in horses: 68 cases (1991-2002). J. Am. Vet. Med. Assoc., v.224, p.562-564, 2004a. 
ARCHER, D.C.; PINCHBECK, G.L.; PROUDMAN, C.J. Factors associated with survival of epiploic foramen entrapment colic: a multicenter, international study. Equine Vet. J., v.43, Suppl.39, p.56-62, 2011.

ARCHER, D.C.; PINCHBECK, G.L.; FRENCH, N.P.; PROUDMAN, C.J. Risk factors for epiploic foramen entrapment colic: an international study. Equine Vet. J., v.40, p.224230, 2008.

ARCHER, D.C.; PROUDMAN, C.J.; PINCHBECK, G. et al. Entrapment of the small intestine in the epiploic foramen in horses: a retrospective analysis of 71 cases recorded between 1991 and 2001. Vet. Rec., v.155, p.793797, 2004b.

BERGEN, T.V.; DOOM, M.; VAN DEN BROECK, W. et al. A topographic anatomical study of the equine epiploic foramen and comparison with laparoscopic visualization. Equine Vet. J., v.47, p.313-318, 2015.

BREIDER, M.A. Endothelium and inflammation. J. Am. Vet. Med. Assoc., v.203, p.300-306, 1993.

CANISSO, I.F.; SOUZA, F.A. Encarceramento do intestino delgado no forame epiplóico em garanhão da raça pantaneiro. Ciênc. Vet. Tróp., v.12, p.60-64, 2009.

ENGELBERT, T.A., TATE, L.P. JR, BOWMAN, K.F.; BRISTOL, D.G. Incarceration of the small intestine in the epiploic foramen: report of 19 cases (1983-1992). Vet. Surg., v.22, p.57-61, 1993.

FREEMAN, D.E. Small Intestine. In: AUER, J.A.; STICK, J.A. Equine surgery. 4.ed. Philadelphia: Saunders, 2012. p.416-453.

FREEMAN, D.E.; PEARN, A.R. Anatomy of the vestibule of the omental bursa and epiploic foramen in the horse. Equine Vet. J., v.47, p.8390, 2015.
FREEMAN, D.E.; SCHAEFFER, D.J. Age distribution of horses with strangulation of the small intestine by a lipoma or in the epiploic foramen: 46 cases (1994-2000). J. Am. Vet. Med. Assoc., v.219, p.87, 2001.

LIVESEY, M.A.; LITTLE, C.B.; BOYD, C. Fatal hemorrhage associated with incarceration of small intestine by the epiploic foramen in three horses. Can. Vet. J., v.32, p.434-436, 1991.

MUELLER, P.O.E; MOORE, J.N, DIVERS, T.J. Gastrointestinal system. In: ORSINI, J.A.; DIVERS, T.J. Equine emergencies: treatment and procedures. 3.ed. St. Louis: Saunders Elsevier, 2008. p.101-188.

SCHMID, A. Die anatomie des foramen epiploicum und seiner benachbarten strukturen und die auswirkungen von alter, rasse und geschlecht auf darmstrangulation durch das foramen epiploicum, inguinalhernie, lipoma pendulans und invagination. 1997. 142f. Thesis (Doctorat in Medicina veterinària) - LudwigMaximilians-Universität, Munich.

VACHON, A.M.; FISCHER, A.T. Small intestinal herniation through the epiploic foramen: 53 cases (1987-1993). Equine Vet. J., v.27, p.373-380, 1995.

WHEAT, J.D. Diseases of the small intestine: diagnosis and treatment. Proc. Am. Assoc. Equine Pract., v.18, p.265-268, 1972.

WICKENS, C.L.; HELESKI, C.R. Crib-biting behavior in horses: a review. Appl. Anim. Behav. Sci., v.128, p.1-9, 2010.

ZINKL, J.G.; MAHENDRA, B.K. Neutrophil function. In: KANEKO, J.J. Clinical biochemistry of domestic animals. 5.ed. San Diego: Academia Press, 2007. p.285-302. 